\title{
Complex Wettability and Coupling Mechanism of Locust Wing
}

\author{
Yan Fang ${ }^{\mathrm{a}}$, Gang Sun ${ }^{\mathrm{b}^{\star}}$, Wanxing Wang ${ }^{\mathrm{c}}$ and Jingshi Yin ${ }^{\mathrm{d}}$ \\ School of Life Science, Changchun Normal University, Changchun 130032, China \\ afangyan124@aliyun.com, bsungang@nenu.edu.cn, c1481399783@qq.com, d634229991@qq.com
}

\begin{abstract}
The complex wettability, chemical composition and microstructure of locust wing surface were investigated by a video-based contact angle (CA) meter, a Fourier transform infrared spectrometer (FT-IR) and a scanning electron microscope (SEM). The wetting mechanism was discussed from the perspective of biological coupling. The locust wing possesses waxy layer and hierarchical micro-morphology. The wing surface is a natural bio-surface with superhydrophobicity (water CA 150.5 158.2 ${ }^{\circ}$ ) and high adhesion, on which the water droplet displays "Gecko state". The special wettability of the wing surface ascribes to the cooperative effect of hydrophobic material and multi-dimensional microstructure. Locust wing can be potentially used as a biomimetic template for design of complex functional surface. This work may bring interesting insights for preparation of micro-controllable superhydrophobic surface and no-loss microfluidic transport channels.
\end{abstract}

Keywords: Wettability, Morphology, Adhesion, Locust, Biomaterial.

\section{Introduction}

The interfacial material with special properties and functions plays a more and more important role in industrial, military, aeronautic, agricultural and domestic fields. Many researchers have focused on lotus leaf, on which a droplet displays a typical Cassie state called "lotus state" (low adhesive superhydrophobicity). However, another special contact state of a droplet on hydrophobic surface called "Gecko state" (high adhesive superhydrophobicity) has attracted less attention. The superhydrophobic material with high adhesion can be used as a "mechanical hand" in the field of no-loss microfluidic transport. We have done some works on microstructure and wettability of butterfly wing surface $[1,2]$. In this work we investigated the chemical composition, microstructure, complex wettability and wetting mechanism of locust wing. The results may bring insights into biomimetic fabrication of micro-controllable interfacial material and novel self-cleaning surface.

\section{Materials and Methods}

Locust specimens of five species (Acrida cinerea, Angaracris rhodopa, Atractomorpha lata, Mecostethus grossus, Primnoa mandshurica) were collected from June to October 2016 in Changchun City, Harbin City and Jilin City, China, and identified by systematic taxonomy. The wings were cleaned, desiccated, and cut into $5 \mathrm{~mm} \times 5 \mathrm{~mm}$ pieces from median intercalary vein of fore wings and remigium of hind wings. A SEM (Hitachi SU8010, Japan) was used for observation and photography of the wings. Using an optical CA measuring system (DataPhysics OCA20, Germany), the CA was measured via sessile drop method. The volume of water droplet was $5 \mu$. The chemical composition of the wing was analyzed by FT-IR (Nicolet FT-IR200, USA). A micro-morphological model for hydrophobicity of the wing was established. Using SPSS 19.0 software, a nonlinear goodness-of-fit test between measured CAs and predicted CAs was carried out.

\section{Results and Discussion}

\subsection{Chemical Composition of the Wing Surface}

All the wing surfaces tested show extremely high similarity in FT-IR spectra. For hind wings, the absorption peaks are at 3272, 2920, 2855, 1638, 1534, 1452, 1233, 1161, and $1077 \mathrm{~cm}^{-1}$ (Fig. 1). The absorption band at $3272 \mathrm{~cm}^{-1}$ results from stretching vibration of $\mathrm{O}-\mathrm{H}, \mathrm{N}-\mathrm{H}$ bases in alcohols, phenols, 
or carboxylic acid. The absorption bands at 2920, $2855 \mathrm{~cm}^{-1}$ result from stretching vibration of C-H base in protein, fat, or chitin. The absorption bands at 1638, 1534, $1452 \mathrm{~cm}^{-1}$ reveal benzene ring in locust wing surface. The absorption bands at 1233, 1161, $1077 \mathrm{~cm}^{-1}$ arise from stretching vibration of C-O base in functional groups such as acid anhydride, acyl chloride, ester, aldehyde, ketone, carboxylic acid, acid amides. The results indicate locust wing is a waxy layer composed mainly of long chain hydrocarbon, tallate and fatty-acid alcohol. The waxy layer, with intrinsic CA of $105^{\circ}$ or so, is the material coupling element for superhydrophobicity of wing surface. The waxy layer alone, however, can not make locust wing surface superhydrophobic.

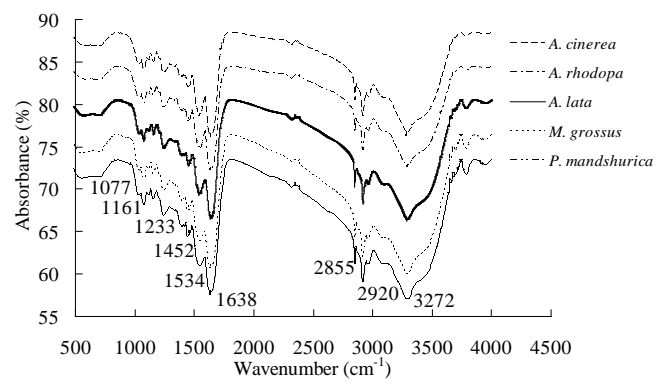

Fig. 1 FT-IR spectra of locust wing surface

\subsection{Micro-morphology of the Wing Surface}

The five locust species have highly similar micro-morphology of wing surface. The wing veins are very clear, constituting the primary microstructure of wing surface [Fig. 2(a)]. The micrometric pillar gibbosities with uniform size distribute regularly and densely in the vein grids, constituting the secondary microstructure of wing surface [Fig. 2(b), 2(c)]. The diameter of gibbosity is $3.6 \sim 8.5 \mu \mathrm{m}$, the height is $4.3 \sim 8.2 \mu \mathrm{m}$, the spacing is $7.9 \sim 15.8 \mu \mathrm{m}$ (Table 1). The numerous nano corrugations between pillar gibbosities constitute the tertiary microstructure of wing surface [Fig. 2(c)].
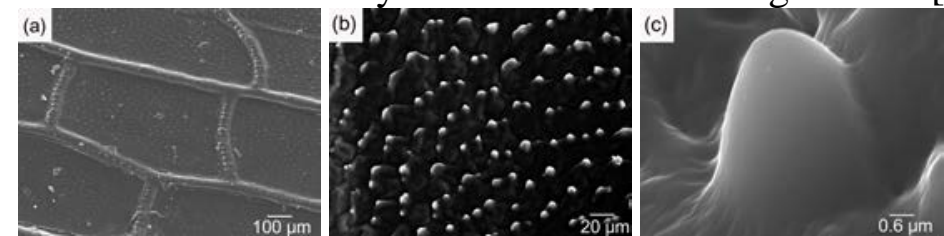

Fig. 2 Hierarchical rough microstructure of locust (A. lata) wing surface. (a) wing vein grids; (b) pillar gibbosities; (c) a single gibbosity and nano corrugations

Table 1 Parameters of the rough micro-morphology (pillar gibbosity) on the locust wing (mean $\pm \mathrm{SD}$ )

\begin{tabular}{|c|c|c|c|c|c|c|c|c|c|c|}
\hline \multirow{2}{*}{ Parameters } & \multicolumn{2}{|c|}{. cinerea } & \multicolumn{2}{|c|}{ A. rhodopa } & \multicolumn{2}{|c|}{ A. Lata } & \multicolumn{2}{|c|}{ M. grossus } & \multicolumn{2}{|c|}{ P. mandshurica } \\
\hline & Fore wing & & & Hind $\mathrm{w}$ & Fore wing & Hind & Fore wing & Hind & & \\
\hline iamete & & & & & $6.0 \pm 0.5$ & & & & & \\
\hline & & & & & & & & & & \\
\hline Spaci & $15.4 \pm 1.3$ & $11.6 \pm 0.8$ & $16.8 \pm 1.5$ & $12.4 \pm 1.1$ & $12.6 \pm 1.3$ & $10.2 \pm 1.0$ & $8.9 \pm 0.8$ & $13.5 \pm 1.4$ & $15.2 \pm 1.4$ & $11.9 \pm 1.3$ \\
\hline
\end{tabular}

\subsection{Superhydrophobicity of the Wing Surface}

The water droplet stands on locust wing surface as a sphere. All the CAs of water droplet on the wing surface of the five locust species are over $150^{\circ}$. The CAs of water droplet on fore wing and hind wing are $150.5 \sim 157.0^{\circ}$ and $152.6 \sim 158.2^{\circ}$, respectively (Table 2), showing that locust wing surface is a natural bio-surface with superhydrophobicity. For the same locust species, hind wing's CA is larger than fore wing's CA, there is significant difference between them $(p<0.05)$. This accords with the functional differentiation of fore wing and hind wing in the process of co-evolution between locust and environment. The fore wing, which is narrow, tough and tensile, mainly takes charge of protection; while the hind wing, which is broad and soft, mainly takes charge of flying. The hind wing requires higher hydrophobicity and self-cleaning performance to reduce flying drag, lighten body burden, and improve movement efficiency. 
Table 2 Measured CAs and predicted CAs on the locust wing surface (mean \pm SD)

\begin{tabular}{|rrrrrrc|}
\hline CA & & A. cinerea & A. rhodopa & A. Lata & M. grossus & P. mandshurica \\
\hline \multirow{2}{*}{ Measured CA $\left({ }^{\circ}\right)$} & Fore wing & $151.3 \pm 3.2$ & $152.2 \pm 2.9$ & $153.7 \pm 3.3$ & $157.0 \pm 4.1$ & $150.5 \pm 3.2$ \\
\cline { 2 - 7 } & Hind wing & $154.6 \pm 4.2$ & $155.8 \pm 3.6$ & $158.2 \pm 3.6$ & $152.6 \pm 3.5$ & $153.4 \pm 3.7$ \\
\hline \multirow{2}{*}{ Predicted CA $\left({ }^{\circ}\right)$} & Fore wing & $152.5 \pm 3.7$ & $155.2 \pm 4.7$ & $155.8 \pm 3.9$ & $157.2 \pm 4.4$ & $153.8 \pm 3.5$ \\
\cline { 2 - 8 } & Hind wing & $156.3 \pm 4.6$ & $158.7 \pm 5.5$ & $159.3 \pm 5.8$ & $150.9 \pm 3.6$ & $157.3 \pm 5.8$ \\
\hline
\end{tabular}

The diameter of a water droplet is about $2.1 \mathrm{~mm}$, far outweighing the spacing $(8.9 \sim 16.8 \mu \mathrm{m})$ of pillar gibbosity. The droplet can neither enter totally the grooves between gibbosities, nor contact fully with wing surface. A gas film exists stably between droplet and wing surface, a composite contact occurs, exhibiting a larger CA. Owing to the chemical composition (waxy layer), locust wing surface can achieve hydrophobicity (CA $105^{\circ}$ or so); owing to the multiple-dimensional rough microstructure, locust wing surface can achieve superhydrophobicity (water CA over $150^{\circ}$ ).

\subsection{High Adhesion of the Wing Surface}

The water droplet displays high adhesion on locust wing surface, and appears as "Gecko state". The droplet does not leave wing surface at any angle of inclination, even verticalized or inverted (Fig. 3). This property resembles that of peanut leaf [3] and rose petal [4]. The most distinct difference between "Gecko state" and "Lotus state" (such as lotus leaf, butterfly wing surface) lies in adhesive performance. Apart from superhydrophobicity, a "Gecko state" droplet exhibits high adhesion and can be pinned on the substrate effectively; whereas a "Lotus state" droplet exhibits low adhesion and extremely small CA hysteresis. When the chemical composition of a solid surface is given, the microtextures play a key role in adhesive performance of droplet on the surface, and determine the wetting contact area and air amount sealed between droplet and surface. On a surface with regularly arraying structures, the bigger the spacing of micrometric structure is and the smaller the density of micrometric structure is, the stronger the adhesive property of droplet is.

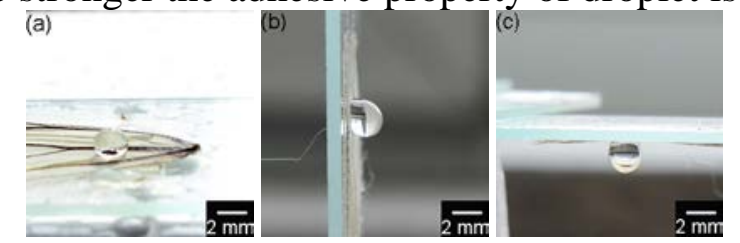

Fig. 3 Contact conditions of water droplet on locust wing. (a) horizontal; (b) vertical; (c) inverted

The wing surfaces of locust and butterfly (both belonging to Class Insecta) show high adhesive superhydrophobicity and low adhesive superhydrophobicity, respectively, which results from the different surface micro-morphology. The spacing between micrometric gibbosities on locust wing surface (averagely $12.85 \mu \mathrm{m}$ ) is 7.4 times of that on butterfly wing surface (averagely $1.74 \mu \mathrm{m}$ ), the density of micrometric gibbosity on locust wing surface is far smaller than that on butterfly wing surface [2]. On locust wing surface, the air fraction (the percentage of liquid/vapor contact area) of water droplet is 0.502 . The micrometric structure of the wing surface can be partially wetted by a water droplet. Relatively less air is trapped and sealed between water droplet and wing surface. As the water droplet is removed from the locust wing surface, negative pressure is produced due to the exchange of confined air, so high adhesive force is induced. On a butterfly (e.g. Polygonia c-aureum) wing surface, the air fraction of water droplet is 0.875 [2], which is much bigger than that on locust wing surface; while the wetting contact area is much smaller than that on a locust wing surface. The water droplet stands on the tips of micrometric gibbosities, much air is left under the droplet. An open air pocket is formed and linked with ambient air. The solid-liquid-gas triple contact lines (TCL) are expected to be contorted and extremely unstable. The butterfly wing surface, on which a water droplet can roll off effortlessly and readily, displays low adhesive superhydrophobicity.

\subsection{Micro-morphological Model for Hydrophobicity of the Wing Surface}

Cassie and Baxter put forward a model to describe the relationship between the apparent CA $\left(\theta_{\mathrm{c}}\right)$ and the intrinsic CA $\left(\theta_{\mathrm{e}}\right)$ when composite contact is formed between a droplet and a rough surface:

$$
\cos \theta_{\mathrm{c}}=\varphi_{\mathrm{s}}\left(1+\cos \theta_{\mathrm{e}}\right)-1
$$

Where $\varphi_{\mathrm{s}}$ is the area fraction of liquid/solid contact $\left(0<\varphi_{\mathrm{s}}<1\right)$. A micro-morphological model for hydrophobicity on locust wing surface is shown in Fig. 4. 


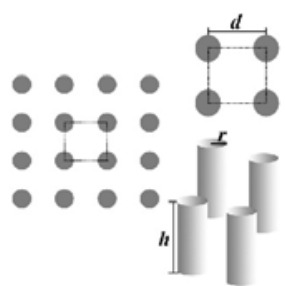

Fig. 4 A micro-morphological model for hydrophobicity on the rough surface of locust wing

The $r, h$ and $d$ represent radius, height and spacing of pillar gibbosity, respectively. Thus,

$$
\varphi_{\mathrm{s}}=\pi r^{2} / d^{2}
$$

$\theta_{\text {c }}$ is determined by $r$ and $d$, has nothing to do with $h$. This is because the droplet does not fill up the rough microstructure completely in a composite contact. Actually, while designing a "Cassie surface", the height $(h)$ of microstructure should be taken into consideration. As the $h$ is bigger, the droplet is more difficult to enter the groove; conversely, in the case of smaller $h$ and larger $d$, the droplet is easier to transit from "Cassie state" with higher energy to "Wenzel state" with lower energy.

The main component of the wing is waxy crystal with an intrinsic CA $\left(\theta_{\mathrm{e}}\right)$ of $105^{\circ}$ or so [2]. $r$ and $d$ values can be known from microstructural parameters on locust wing surface (Table 1$), \varphi_{\mathrm{s}}$ can be calculated and substituted into Eq. (1), then the predicted CA $\left(\theta_{\mathrm{c}}\right)$ can be obtained (Table 2). The $R_{\text {New }}$ values (the coefficient of determination in nonlinear goodness-of-fit test) are $0.934 \sim 0.968$ for fore wing, 0.928 0.956 for hind wing. The degree of fitting is fine, demonstrating the micro-morphological model for hydrophobicity is in good accord with Cassie equation.

\section{Conclusions}

Locust wing surface possesses multiple-dimensional rough microstructures and waxy layer. Composite contact occurs between droplet and locust wing. Locust wing surface is a natural bio-surface with superhydrophobicity (water CA 150.5 158.2 ${ }^{\circ}$ ) and high adhesion. The micro-morphological model for hydrophobicity of locust wing established here is in good accord with Cassie equation, the degree of fitting between predicted CAs and measured CAs is fine. The complex wettability of locust wing is attributed to cooperative effect of hydrophobic material and hierarchical microstructure. Locust wing can be potentially used as a biomimetic template for design of functional surface with special wettability. This work may bring interesting insights for preparation of micro-controllable superhydrophobic surface and no-loss microfluidic transport channels.

\section{Acknowledgments}

This work was financially supported by the National Natural Science Foundation of China (Grant No. 31671010) and the Innovative Program for Postgraduates of Changchun Normal University (Grant No. cscxy2017003, cscxy2017006). Dr. Prof. Gang Sun is the corresponding author of this paper.

\section{References}

[1] G. Sun, Y. Fang, Q. Cong, L.Q. Ren, Anisotropism of the non-smooth surface of butterfly wing, J. Bion. Eng. 6 (2009) 71-76.

[2] Y. Fang, G. Sun, T.Q. Wang, Q. Cong, Hydrophobicity mechanism of non-smooth pattern on surface of butterfly wing, Chin. Sci. Bull. 52 (2007) 711-716.

[3] S. Yang, J. Ju, Y.C. Qiu, Superhydrophobic materials: Peanut leaf inspired multifunctional surfaces, Small 10 (2014) 214-214.

[4] L. Feng, Y.A. Zhang, J.M. Xi, Petal effect: A superhydrophobic state with high adhesive force, Langmuir 24 (2008) 4114-4119. 